\title{
An Infrastructure for Interactive and Playful Learning in Augmented Toy Environments
}

\author{
Steve Hinske ${ }^{1}$, Marc Langheinrich ${ }^{2}$ \\ ${ }^{1}$ Institute for Pervasive Computing, ETH Zurich, Switzerland, steve.hinske@inf.ethz.ch \\ ${ }^{2}$ Faculty of Informatics, University of Lugano (USI), Switzerland, langheinrich@acm.org
}

\begin{abstract}
In this paper we describe our approach to realize a pervasive (computing) learning environment for children based on augmented toys: traditional toys that are equipped with pervasive computing technologies and can thus be linked with virtual information such as educational content. We report on our experiences building and deploying the Augmented Knight's Castle, an augmented toy environment that allows for learning about the Middle Ages in a playful way. We demonstrate and discuss how educational content is provided in such an environment and describe the design of the underlying infrastructure.
\end{abstract}

\section{Introduction}

Augmented toys have the potential to combine the best of two worlds: the real, physical world and the virtual world, respectively. While traditional toys allow for socializing and the benefits of tactile sensation, they are limited in their capabilities of providing the players with sensory immersion and support for complex interactions. Virtual toys (i.e., video games) on the other hand, are able to design fully artificial worlds using elements of graphic novels or movies that can individually interact with the players, yet they typically lack haptic feedback and often limit social face-to-face interaction with other players. Adding virtual elements to traditional toys promises to combine these two paradigms to their respective advantage, thus resulting in engaging augmented play environments (ATEs).

This augmentation does not only contribute to the play experience, but can also enable the integration of educational content and help conveying it in a playful manner. To achieve this, however, an infrastructure must be provided that takes into account the very dynamic nature of such play environments, without compromising the traditional play experience, i.e., the technology required to integrate the educational content should merely be an add-on and not become the sole focus of the play environment [2].

This paper presents an ATE that enables interactive and playful learning for children. Resembling a medieval world, the figures in this play scenario can tell the children about their lives and roles and provide them with generally interesting facts about the Middle Ages. We developed an infrastructure for the creation, modification, and integration of educational content for this ATE. The central idea is that each (play) object has a virtual counterpart that can be linked with a variety of educational information, which is then made available in the play environment in an unobtrusive and playful way. Principally, the educational content can be provided twofold:

1. Directly, using audio feedback (i.e., verbal commentaries by the figures).

2. Indirectly, through a mediator such as a cell phone or a computer display.

The underlying infrastructure features a number of different interfaces to account for the capabilities and idiosyncrasies of the different end-user devices, while using a unified representation of the educational content that is independent of the actual feedback channel used.

The paper is structured as follows: first, we introduce our particular ATE and its operation, emphasizing the provision of educational content. Second, we describe the infrastructure necessary for such a pervasive (computing) learning environment. Finally, a summary and a discussion of the findings conclude this paper.

\section{Playful Learning with the Augmented Knight's Castle}

The Augmented Knight's Castle (AKC) is an ATE resembling a medieval world (see Fig. 1). Employing pervasive computing technologies such as radio frequency identification (RFID) technology, the AKC is a traditional Playmobil Middle Ages play set that is 
capable of detecting the figures as they are placed on and moved within the game environment, and reacting accordingly [5].

We utilize the location information of individual figures to provide them with custom sound effects and verbal commentaries, based on their current and past locations. Additional effects such as light or smoke further enrich the play experience of the children and let the play set come alive. For example, if the dragon is taken out from the dungeon beneath the dragon tower, its exiting is accompanied by red light, smoke, and a roaring sound.

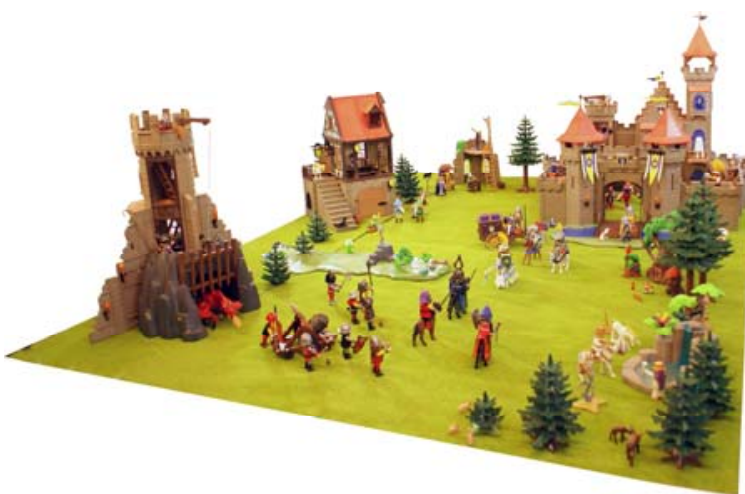

Figure 1: A scene from the AKC.

For the scope of this paper, we will concentrate on the audio output, especially on the verbal commentaries of the figures as they can convey educational content in a playful way by engaging children in a story [6]. To maximize the integration of the learning experiences in the children's play, the figures tell about facts and tales from their perspective, e.g., the blacksmith tells about how armors and weapons were forged while the king talks about his days at the court. They can also address the children directly, thus stimulating a direct interaction between them and the children, e.g., the bard starts singing and invites the children to sing along.
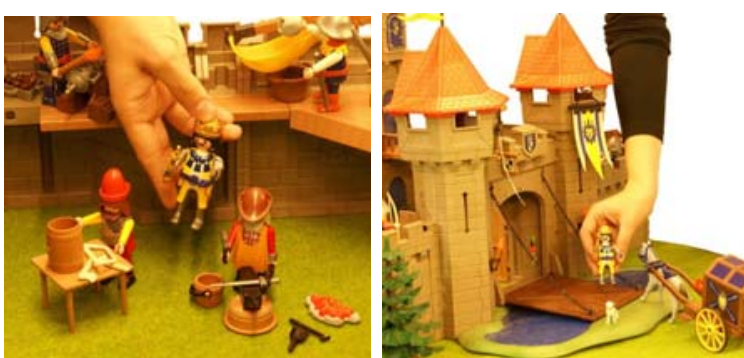

Fig. 2. The king explains details about the life in a castle to the child
Even more complex scenarios are possible: the king explains what it is like to live with his family and court in a castle in the Middle Ages, including different topics, e.g., life in a castle, the royal family, the role of craftsmen and guilds, weaponry or heraldry. As shown in Fig. 2, the king invites the child to follow him through different areas and settings of the castle. The king explains for each setting (e.g., defense weaponry, craftsmen and drawbridge) different facts and asks the child to place him into different locations or to leave him at the current location in order to explain more details.

While the verbal commentaries played by the AKC can be used to convey facts about the Middle Ages in a playful way, some educational content may benefit from further multimedia capabilities such as images or videos. As the AKC does not feature any displays they would counteract the traditional play atmosphere - we support the inclusion of additional devices such as displays or personal digital assistants (PDAs) into the game environment. In particular, we integrated a magic mirror (a PC display with a touch screen) and a loupe (a PDA) into the game environment.

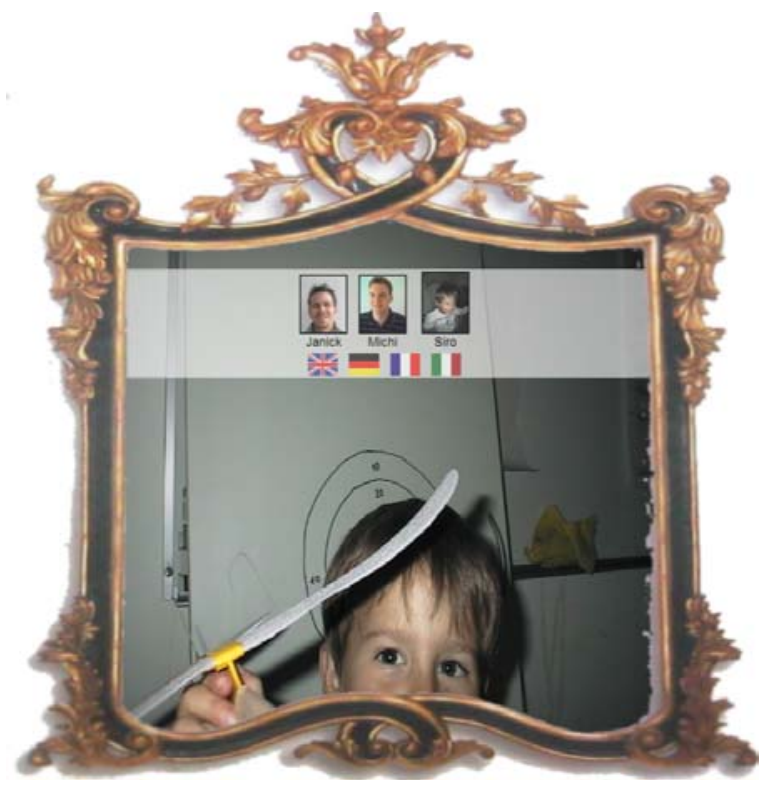

Figure 3: The login screen of the magic mirror.

Our main concern was that the devices used by the children would smoothly integrate in the play environment and not disrupt the children's play experience. Our magic mirror, e.g., is modeled after its counterpart in "Snow White and the Seven Dwarfs," where it represents an all-knowing source of information. Matching our medieval world and given the respondent function the device is supposed to play, we deemed it a good choice (also cf. [7]). 
The magic mirror consists of a PC with a display, a web cam, and a small wooden pedestal with an embedded RFID antenna. A child can use the magic mirror by simply placing a figure on the pedestal: the mirror will then switch from displaying the child's reflection (using the web cam) to displaying the learning modules associated with this figure (see Fig. 3 and 4). In order to recognize the child, we provide them with a personal magic item (e.g., a brooch, a magic card, or a figurine) that they will need to wear or place next to the mirror in order to activate it. Each magic item contains an RFID transponder that identifies the child in the game environment, thus supporting an individual learning history for each child in order to keep track of his or her progress.

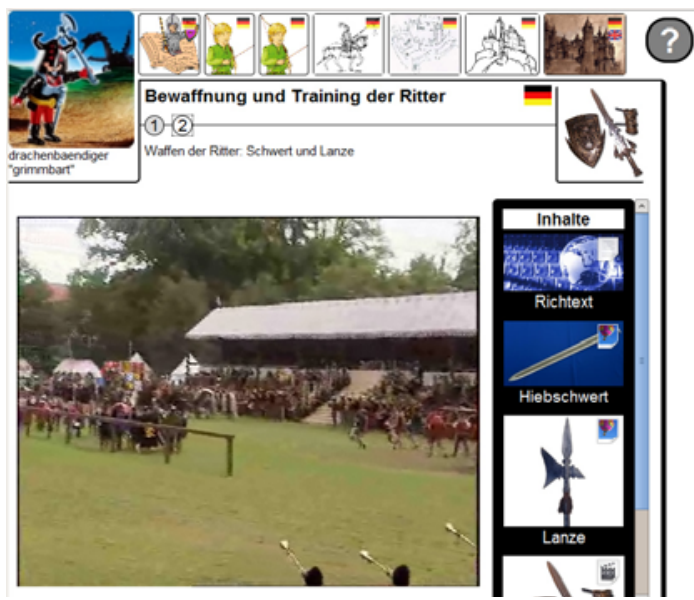

Figure 4: A screenshot of the magic mirror.

The magic mirror then displays the educational content available for this play figure. Depending on the available content, the children can select from a number of different learning modules and retrieve information about the figure as well as facts about the Middle Ages in general. In contrast to the verbal commentaries, the magic mirror is much more powerful in terms of feedback (i.e., text, pictures, and videos).

The main advantage, however, is the higher level of interactivity: while the verbal commentaries during the play allow for some interaction with the children (cf. Fig. 2), the magic mirror is capable of more sophisticated selection and feedback processes such as quizzes and puzzles with regard to the previously displayed educational content.

In addition to the magic mirror, a PDA-based solution was implemented. This approach is mainly motivated by two trends regarding mobile phones and similar devices: first, these devices are steadily becoming more powerful with novel capabilities being added and old ones improved constantly. Second, the number of children in possession of mobile phones is continuously growing, even at elementary school age [3]. In other words, small mobile devices must be considered seriously when designing pervasive (computing) environments, even for children.

The PDA can be seen as a pocket magic mirror and principally offers the same functionality as the magic mirror. The problem is that a PDA has limited resources by comparison and a standard browser on the PDA is often not sufficient since the browser usually displays the original website with scroll bars, necessitating a custom-built user interface. We thus implemented our own user interface with Microsoft Visual Studio .NET. The PDA, an HP iPAQ hx2400, has integrated $\mathrm{WiFi}$ and an attached RFID reader (Socket RSC 6E) (see Fig. 5).

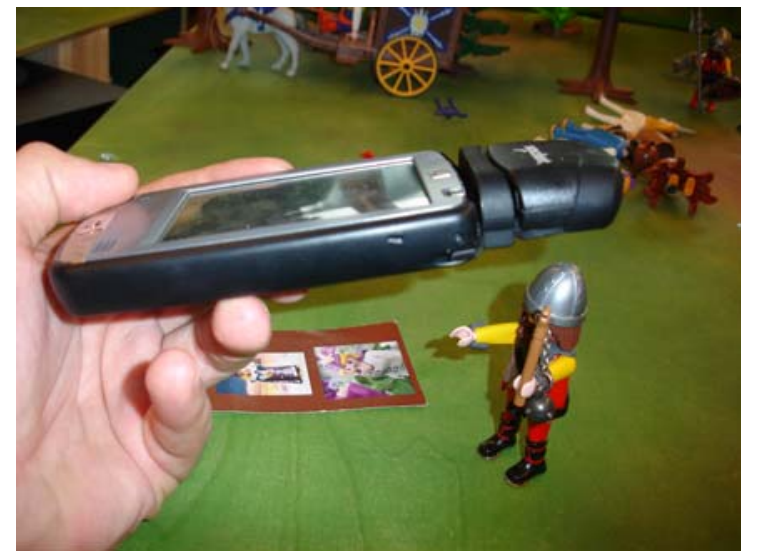

Figure 5: The PDA with the attached RFID.

This device is capable of (dis-)playing of almost all learning modules - the multimedia content is dynamically adjusted to the $\mathrm{I} / \mathrm{O}$ capabilities of this device (e.g., images /videos are resized accordingly).

\section{The Underlying Infrastructure}

Our main goal was to design a flexible, extensible, and easily comprehensible infrastructure for interactive and playful learning in augmented toy environments. To this end, the infrastructure must provide means to easily link educational content to play objects and means to retrieve this content.

While our main target group are children playing with the play set, there are two more parties involved: first, parents or educators (i.e., pre-school teachers), who supposedly purchase the toys and have an interest in knowing what these toys can and cannot do. Second, there are the content and toy designers, who create educational content modules and subsequently associate them with the play objects. With regard to the augmented toy environment and the individual interests, we can derive the following use cases: 
- Children can interact with the AKC and retrieve educational content using the magic mirror or the PDA.

- Parents / educators can selectively activate educational content for the children. For them, a web-based user interface is provided. Furthermore, they can review each child's individual learning history and progress (i.e., what modules the child has viewed, what quizzes have been solved, etc).

- Developers can create and modify learning modules and integrate multimedia content using a content management system.

Central to the infrastructure are the play objects and the associated educational content. The educational content is delivered in learning modules with each module representing a complete and closed topic. Each module is divided into several, consecutive levels, which reflect the gradually increasing complexity and difficulty of the content. Each level is associated with multimedia content (see ER diagram in Fig. 6).

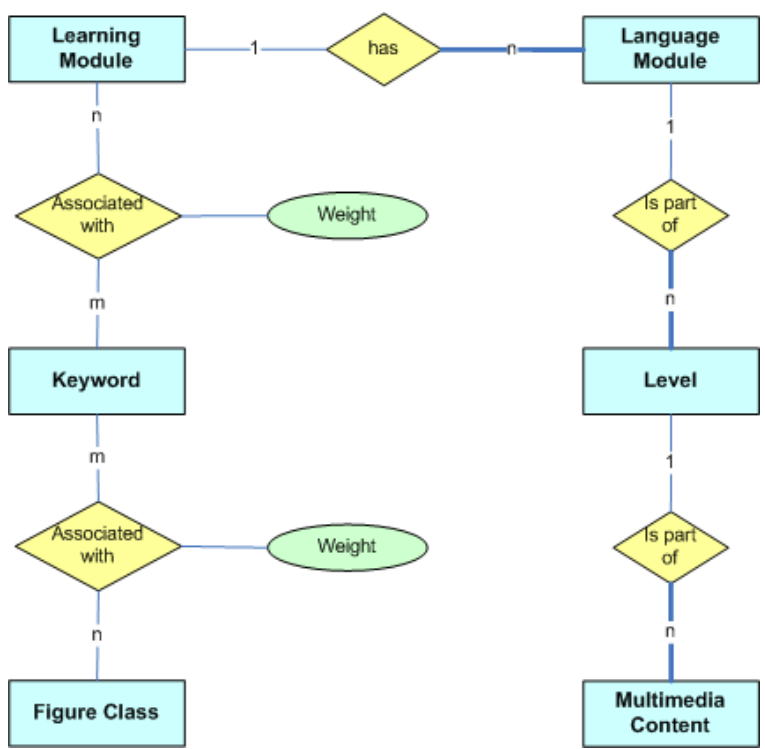

Figure 6: First part of the entity-relationship diagram.

A child can select any level of a module that is unlocked. While most levels of a module are available from the beginning, a level can have a quiz that must be solved in order to proceed to the next level (unlock); these levels cannot be skipped. This not only ensures a revisable learning progress but is even necessary when a higher level is dependent on the information provided in previous levels. In addition to this, each module might be available in several languages. Parents and educators configure what languages should be available, and the children can switch between these selected languages by simply pressing a button. The presentation of the same educational content in different languages can help the children with learning foreign languages in a playful manner.

The modules are associated with play objects using keywords. To this end, each figure and each learning module has a set of keywords associated with it. The keywords are meant to describe them as precisely as possible. They are assigned by the designers of the figures and the developers of the learning modules, respectively. The keywords can be labeled sensitive by the designer if the content is potentially critical (e.g., learning modules about weapons, torturing methods, sexuality, etc.) and also have a minimum age attribute. Additionally, the keywords can be weighed to reflect the relevance for a figure or learning module.

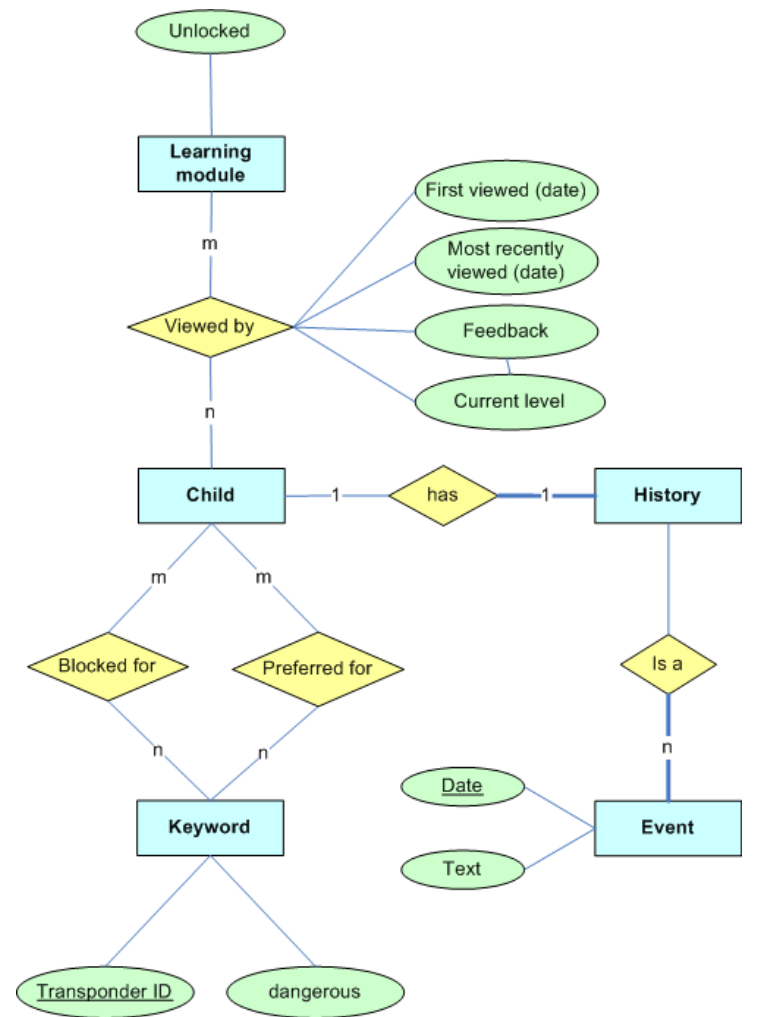

Figure 7: Second part of the ER diagram.

A learning module is relevant for a figure, if one or more keywords match. This approach has several advantages compared to fixed associations:

- There can be many designers of educational content and figures; there is no synchronization or coordination required.

- Newly created learning modules can be easily associated with figures already in existence.

- The number of learning modules is not limited; for each figure there is potentially a great variety of different modules to pick from. 
It is even possible to easily link different learning modules, which allows for a more in-depth learning experience.

Educators can pre-configure the selection process by marking keywords for a particular child as blocked (i.e., modules that contain at least one blocked keyword are exempt from being displayed) or preferred to exclusively select modules for a child (i.e., modules must contain at least one preferred keyword to appear on the list) (see Fig. 7). The block attribute is stronger than preferred: if a learning module contains both, keywords marked as blocked and keywords marked as preferred, the module is not available.

Conclusively, a learning module is not available for a child if at least one of the following conditions is true:

- The module is not available in the currently selected language.

- The minimum age of the module is higher than the child's age.

- The module contains one or more keywords that are blocked by the parents.

To access a learning module, the child uses one of the available interfaces (in our case the magic mirror or the loupe) and scans a play figure (Fig. 8 shows a sequence diagram of the magic mirror). The device then provides a list with all associated modules sorted by their relevance. The relevance is determined by the ranking value $\mathcal{R}$ of a module $m \in M$ for any given figure $f$ :

$$
m \in M: \mathcal{R}(m, f)=\sum_{t \in T} G(m, k) * G(f, k)
$$

with $G(m, k)$ being the weight of the keyword $k$ associated to the module $m$ and $G(f, k)$ being the weight of the keyword assigned to figure $f$. Accordingly, the maximal ranking value $\mathcal{R}_{M A X}$ of a module $m$ is

$$
m \in M: \mathcal{R}_{M A X}=\max _{m \in M}(\mathcal{R}(m, f))
$$

These values indicate how well a module matches a figure. The ranking values are sorted from high to low. In addition to that, a selection value $\mathcal{S}$ is used to select only modules with a higher ranking value than $\mathcal{S}$. In other words, the lower the value $\mathcal{S}$, the more modules are being displayed.

The modules are divided into two groups: the first group contains all learning modules with at least one keyword marked as preferred and the second group all remaining modules. In both groups the learning modules are then sorted by their ranking value. The subsequent merging of both groups by appending the second one to the end of the first one results in the final list of available learning modules sorted by their relevance for this child.

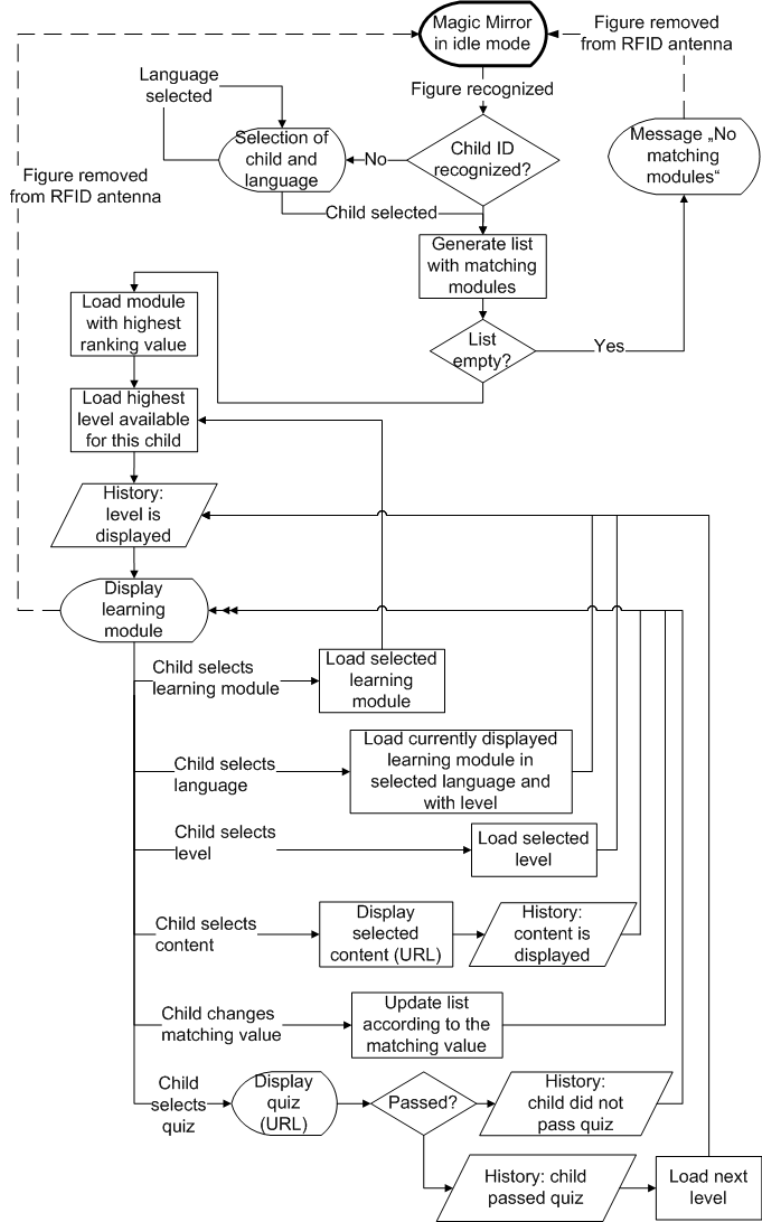

Figure 8: The sequence diagram of the magic mirror.

To guarantee maximum flexibility, the multimedia content is referenced by a uniform resource locator (URL): on the one hand, the educational content is globally accessible and does not need to be stored locally (i.e., the educational content is always up-todate), and, on the other hand, the educational content can be provided using numerous different technologies. The same applies to the quizzes.

The infrastructure is based on web services to ensure maximal flexibility with regard to the end-user device. This is required as not all devices are capable of displaying websites, or the (small) screen of the device necessitates adjusting the provided content to fit the output capabilities of the device.

\section{Discussion and Conclusions}

In this paper we introduced an ATE for interactive and playful learning. The educational content can be delivered in two ways, directly (i.e., the figures tell stories during the play) and indirectly (i.e., the children 
use the magic mirror or the loupe). We presented an infrastructure for linking educational content to the figures, which can then provide information and tell stories to the children. The AKC can thus be seen as good example of situated learning [9]: the children learn about the world (here, the Middle Ages) while playing and they can also use the comments and facts to further embellish their own stories.

While our focus is on learning, the infrastructure is by no means limited to educational content: the flexible design allows potentially integrating all kinds of information. Matter-of-factly, the idea is to map all information relevant for an object to it, which also includes, for example, manufacturing and shipping information (e.g., the object could "tell" where and when it was created and how it was transported from its place of manufacture to the current location).

One of the central research questions with regard to pervasive learning is "how can pervasive technologies be used to support new ways of learning about different educational subject matters?" [8]. We think that research such as the presented project brings us closer to answering this question: having play figures telling the children about facts and stories as well engaging them in interactive learning without compromising the original play setting is certainly a "new way".

To support this claim, we conducted a user study with over one hundred children in an elementary school in Germany [3]. The children played with both with the AKC as well as with a non-augmented version of the castle for approximately 35 minutes, followed by group interviews with the researchers. During the play with the AKC, game figures would talk about their lives in the Middle Ages. Both directly after the play session, as well as two months later, children were then asked a few questions about life in the Middle Ages, in order to assess what knowledge they retained. The analysis showed that children were very susceptible for educational content conveyed in a playful way, and that this content was retained especially well among the slightly older children (aged 9-11).

However, as Kurti et al. point out [8], “designing technology support for situated learning is a challenging task, since in many cases technology tends to shift the learning environment to a more computerbased representation moving away from the core ideas of situated learning $[1,4]$ ". They continue: "However, pervasive computing opens new dimensions to avoid this diversion, by providing means to trustfully representation of learners' contexts by placing them back into the authentic. Pervasive environments provide the possibilities of embedding computational support for the learning activity in the learner's physical and social contexts [10].”
The AKC in its basic version (without mediators) definitely complies with these requirements (see also [2]). Even the mediators, the magic mirror and the PDA-based loupe, respectively, are designed and integrated to not distract children from their original playing but they rather serve as information terminals that can be consulted when desired, thus contributing to the idea of offering the information whenever and wherever needed without disrupting the natural environment of the user.

\section{References}

[1] Herrington, J., Oliver, R., "Critical characteristics of situated learning: Implications for the instructional design of multimedia”, In: Pearce, J., Ellis, A. (Eds.), Learning with technology. Parkville, Vic: University of Melbourne, 1995, pp. 235-262.

[2] Hinske, S., Langheinrich, M., Lampe, M., "Towards Guidelines for Designing Augmented Toy Environments”, In: v.d.Schijff, J., Marsden, G., Kotze, P. (eds.), Proc. of the 6th ACM Conference on Designing Interactive Systems (DIS 2008), ACM Press, New York, NY, USA, February 2008.

[3] Hinske, S., Lampe, M., Price, S., Yuill, N., Langheinrich, M., "Kingdom of the Knights: Evaluation of An Augmented Toy Environment for Storytelling and Playful Learning", submitted for publication, 2008.

[4] Hummel, H.G.K., "Distance Education and situated learning: Paradox or Partnership", Educational Technology, Vol. 33, No. 12, 1993, pp. 11-22.

[5] Lampe, M., Hinske, S., “The Augmented Knight’s Castle - Integrating Pervasive and Mobile Computing Technologies into Traditional Toy Environments”, In: Magerkurth, C., Röcker, C. (eds.): A Reader for Pervasive Gaming Research. Vol. 1, Shaker Verlag, 2007.

[6] Lampe, M., Hinske, S., "Integrating Interactive Learning Experiences into Augmented Toy Environments", Proc. of Pervasive Learning Workshop at Pervasive 2007.

[7] Kuniavsky, M., "Magic as a Metaphor for Ubiqitous Computing”, Ambidextrous, Vol. 6, 2007, pp. 36-37.

[8] Kurti, A., Spikol, D., Milrad, M., Svensson, M. Pettersson, O., "Exploring How Pervasive Computing Can Support Situated Learning", Proc. of Pervasive Learning Workshop at Pervasive 2007. Toronto, Canada, May 2007

[9] Lave, J., Wenger, E., "Situated Learning: Legitimate Peripheral Participation”, Cambridge, Cambridge University Press, 1991

[10] Lyytinen, K., Yoo, Y., "Issues and Challenges in Ubiquitous Computing 2003”, Communications of ACM, Vol. 45, No. 12, 2002, pp. 62-65. 